IAC-12-D1.4.10

\title{
STUDY ON THE ORBITAL MANEUVERING CAPABILITY OF H-2A KICK STAGE
}

\author{
Toshinori Ikenaga \\ Japan Aerospace Exploration Agency, Japan, ikenaga.toshinori@jaxa.jp \\ Masayoshi Utashima ${ }^{* 1}$, Nobuaki Ishii ${ }^{* 2}$, Tetsuo Hiraiwa ${ }^{* 3}$ and Atsushi Noda ${ }^{* 4}$
}

This paper describes a concept study of an optional kick stage system named "PLUS (for Planetary mission, Long-duration small-thrust Upper Stage)" equipped with a relatively small-thrust engine for interplanetary missions. The thrust force of PLUS assumed in this study is a maximum $29.4 \mathrm{kN}$, which is relatively small, used to inject a payload into interplanetary orbit with sufficiently low gravity loss i.e. less than $2 \%$, although two split delta-Vs such as the Russian Proton/Breeze-M will improve performance efficiency ${ }^{1}$. The orbital maneuvering capability of PLUS system is evaluated through Mars orbital maneuvering simulations. In this study, two types of PLUS systems are assumed: First, a $29.4 \mathrm{kN}$ thrust force kicks stage system designated as "PLUS1" to inject the $3000 \mathrm{~kg}$ main payload into Mars transfer orbit. Second, a $9.8 \mathrm{kN}$ thrust force small PLUS system designated as "PLUS2" to inject a $500 \mathrm{~kg}$ secondary payload into Mars transfer orbit. In the first case, the above-mentioned split delta-Vs are conducted to inject the main payload into Mars transfer orbit with sufficiently low gravity loss. In the second case, on the other hand, PLUS2 attached to the secondary payload is dual launched together with a primary payload into a geostationary transfer orbit, GTO, whereupon PLUS2 is initiated slightly before perigee to inject the secondary payload into Mars transfer orbit utilizing Electric delta-V Earth Gravity Assist, EDVEGA scheme via the on-board Ion Electric propulsion System, IES ${ }^{2,3}$. Throughout the simulations, some optimized configurations of PLUS system covering a wide variety of space missions are suggested in this paper.

\section{INTRODUCTION}

The main purpose of this study is to evaluate the launch capability of an optional kick stage system named "PLUS (for Planetary mission, Long-duration small-thrust Upper Stage)" equipped with a LOX/ethanol engine, which is under development in the Kakuda space center of the Japan Aerospace Exploration Agency, JAXA, from an interplanetary mission i.e. Mars mission perspective.

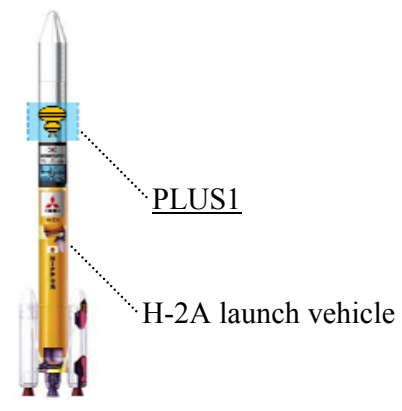

Figure 1. Launch System Configuration of Case 1

In this study, two different types of PLUS systems are assumed: First, a $29.4 \mathrm{kN}$ thrust force kick stage system designated as "PLUS1" to inject a $3000 \mathrm{~kg}$ main payload into Mars transfer orbit. Second, a $9.8 \mathrm{kN}$ thrust force small PLUS system designated as "PLUS2" to inject a $500 \mathrm{~kg}$ secondary payload into Mars transfer orbit.

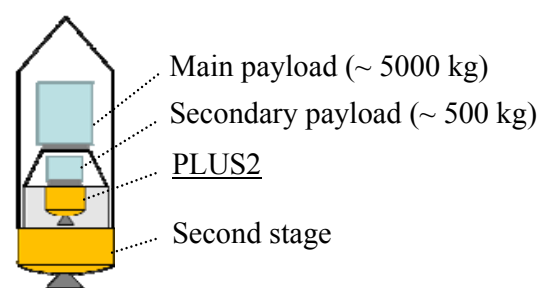

Figure 2. Launch System Configuration of Case 2

In the first case, designated as "Case 1" in this study, PLUS1 is assumed to be optionally attached to the Japanese flagship launch system, H-2A. Figure 1 shows the launch system configuration of "Case 1". In this case, the main payload and PLUS1 are injected into a $300 \mathrm{~km}$ altitude circular parking orbit via the first and second stages of H-2A, whereupon PLUS1 is fired to inject the main payload into Mars transfer orbit. However, since the thrust force of the assumed PLUS1 is relatively small for such use, two split delta-Vs, such as the Russian Proton/Breeze-M, are applied to improve

\footnotetext{
${ }^{{ }^{1} 1}$ Japan Aerospace Exploration Agency, Japan, utashima.masayoshi@jaxa.jp

*2 Japan Aerospace Exploration Agency, Japan, ishii.nobuaki@jaxa.jp

*3 Japan Aerospace Exploration Agency, Japan, hiraiwa.tetsuo@jaxa.jp

${ }^{* 4}$ Japan Aerospace Exploration Agency, Japan, noda.atsushi@jaxa.jp
} 
the performance efficiency of the above-mentioned PLUS1 system. Gravity loss is focused on to measure the performance efficiency of the low-cost and smallthrust PLUS system.

In the second case, designated as "Case 2", PLUS2 attached to the secondary payload is dual launched together with a primary payload into a geostationary transfer orbit, GTO, whereupon PLUS2 is initiated at perigee to inject the secondary payload into the energy garnering orbit, EGO. Figure 2 shows the launch system configuration of Case 2.

Although the relative velocity at Earth departure is almost zero, the on-board IES accelerates the spacecraft through the EDVEGA scheme, which increases the Earth relative velocity at the Earth re-encounter point up to about $2.7 \mathrm{~km} / \mathrm{sec}$; enabling the spacecraft to inject into Mars transfer orbit after the Earth gravity assist.

Throughout the simulations, some optimized configurations of PLUS system covering a wide variety of space missions are suggested in this paper.

\section{$\underline{\text { II. FORCE MODEL AND LAUNCH WINDOW }}$}

\section{II.I Force Model}

In this study, the gravitational forces of the Sun and Earth are considered. It is assumed that the orbits of the Earth and the destination planet i.e. Mars lie on the ecliptic plane. Conversely, the eccentricities of both orbits are considered.

\section{II.II Launch Window}

The transfer orbit between the Earth and Mars is determined by solving Lambert's problem. The outbound relative velocity at the Earth departure, designated as $V_{\infty}$, is also determined by the transfer orbit. In practice, the $\Delta \mathrm{V}$ generated by a launch vehicle is limited and the lower $\Delta \mathrm{V}$ the better.

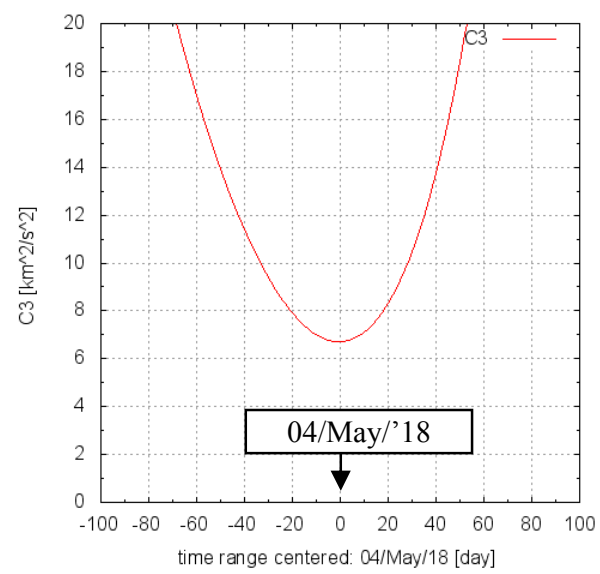

Figure 3. Departure Epoch and $C_{3}$

In this study, May $4^{\text {th }}, 2018$ is determined as the departure epoch to Mars. Figure 3 shows the relationship between the launch epoch and orbital energy $C_{3}$, which is the square of the out-bound relative velocity at the Earth departure. The time of flight for the transfer is 200 days.

\section{ORBITAL MANEUVER}

\section{III.I Orbital Maneuver of Case 1}

In Case 1, the main payload and PLUS1 are initially injected into a $300 \mathrm{~km}$ altitude circular parking orbit. The PLUS1 is initiated at the optimal point to inject the payload into Mars transfer orbit.

Generally, a kick stage is fired only once, designated as "1-Burn" in this paper, and the spacecraft is injected into the transfer orbit. However the thrust force assumed in this study is relatively small i.e. $29.4 \mathrm{kN}$, hence split delta-Vs, designated as "2-Burn", are applied and the performance efficiency, i.e. gravity losses of both cases, are compared. Figure 4 shows the orbital maneuvers in the cases of "1-Burn" and "2-Burn".

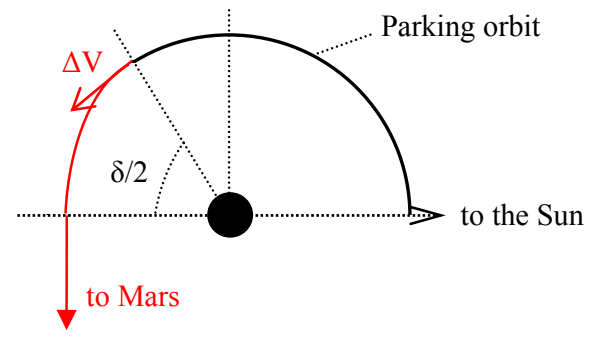

a) 1-Burn

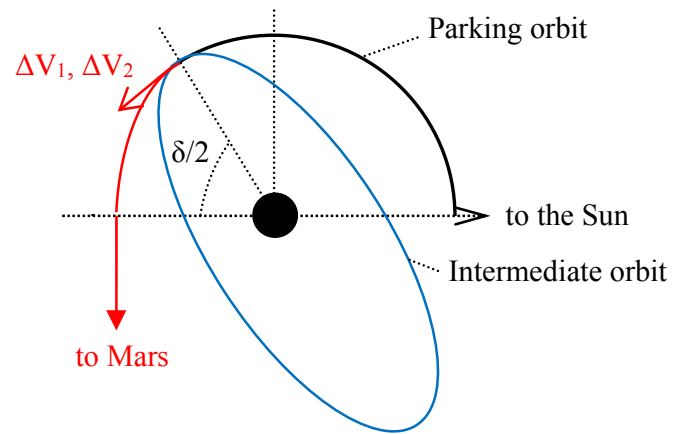

b) 2-Burn

Figure 4. Diagram of Orbital Maneuver of Case 1

The angle $\delta / 2$, which is the angle between the "impulsive" $\Delta \mathrm{V}$ start point and the Sun-Earth line, is described as the following equation:

$$
\sin \left(\frac{\delta}{2}\right)=\frac{1}{1+\Psi}
$$

where; 


$$
\Psi \equiv\left(\frac{V_{\infty}}{V_{p}}\right)^{2}
$$

$V_{\infty}$ is the out-bound Earth relative velocity to Mars, which is determined by Lambert's theorem, and $V_{p}$ is the orbital velocity of $300 \mathrm{~km}$ altitude circular parking orbit. In this study, the value of $V_{\infty}$ is $2.6 \mathrm{~km} / \mathrm{s}$ and $V_{p}$ is $7.7 \mathrm{~km} / \mathrm{s}$, hence the turning angle $\delta / 2$ is $64.0 \mathrm{deg}$.

The main purpose of the Case 1 study is to evaluate the gravity loss of PLUS1, hence finite time thrust is simulated. Figure 5 shows a diagram of the finite thrust simulation.

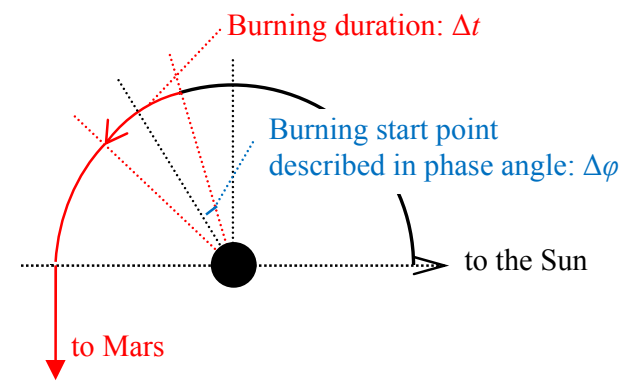

Figure 5. Diagram of Finite Time Thrust

Here, the mass of propellant $m_{p}[\mathrm{~kg}]$ consumed in the burning duration, is described in the following;

$$
m_{p}=|\dot{m}| \Delta t
$$

where $\dot{m}[\mathrm{~kg} / \mathrm{sec}]$ is the mass flow rate which is defined as:

$$
|\dot{m}|=\frac{F}{g I_{s p}}
$$

where $F[\mathrm{~N}]$ is the thrust force, $g$ is the standard Earth's gravitational acceleration i.e. $9.80665 \mathrm{~m} / \mathrm{sec}^{2}$, and $I_{s p}$ [sec] is the specific impulse.

The velocity change, $\Delta \mathrm{V}$, produced by the propellant $m_{p}$ is described as the rocket equation';

$$
\Delta V=g I_{s p} \ln \left(\frac{m_{0}}{m_{0}-m_{p}}\right)
$$

where $m_{0}[\mathrm{~kg}]$ is the initial mass of PLUS system including the payload. From Eqs. (5), $m_{p}$ is also described as the follows;

$$
m_{p}=m_{0}\left(1-\exp \left(-\frac{\Delta V_{e s c}}{g I_{s p}}\right)\right)
$$

where $\Delta V_{\text {esc }}[\mathrm{m} / \mathrm{s}]$ is the $\Delta \mathrm{V}$ required to inject the spacecraft into Mars transfer orbit from the parking orbit. $\Delta V_{e s c}$ is described as the following;

$$
\Delta V_{e s c}=\sqrt{V_{\infty}^{2}+\frac{2 \mu_{E}}{r_{p}}}-\sqrt{\frac{\mu_{E}}{r_{p}}}
$$

where $\mu_{E}$ is the Earth's gravitational constant $3.986004418 \times 10^{14} \mathrm{~m}^{3} / \mathrm{sec}^{2}$ and $r_{p}[\mathrm{~m}]$ is the radius of the parking orbit. As mentioned, $V_{\text {esc }}[\mathrm{m} / \mathrm{s}]$ is determined by solving Lambert's problem.

From the Eqs. (3), Eqs. (4) and Eqs. (6) then the burning duration $\Delta t$ is described as the following:

$$
\Delta t=\frac{m_{0}}{F} g I_{s p}\left(1-\exp \left(-\frac{\Delta V_{e s c}}{g I_{s p}}\right)\right)
$$

The burning start point is described in phase angle $\Delta \varphi$. The $\Delta \mathrm{V}$ starts at the point angle $\Delta \varphi$ earlier from the impulsive $\Delta \mathrm{V}$ point. The phase angle $\Delta \varphi$ is described in the following:

$$
\Delta \varphi=\frac{\Delta t}{2} \sqrt{\frac{\mu_{E}}{a^{3}}}
$$

where $a[\mathrm{~m}]$ is the semi-major axis of the orbit i.e. the "circular" parking orbit, or "highly elliptical" intermediate orbit.

\section{III.II Orbital Maneuver of Case 2}

In Case 2, the primary payload and secondary payload attached to PLUS2 are dual launched into GTO. The launch epoch and apsis line of the GTO are determined by the mission requirement of the primary payload rather than secondary payload.

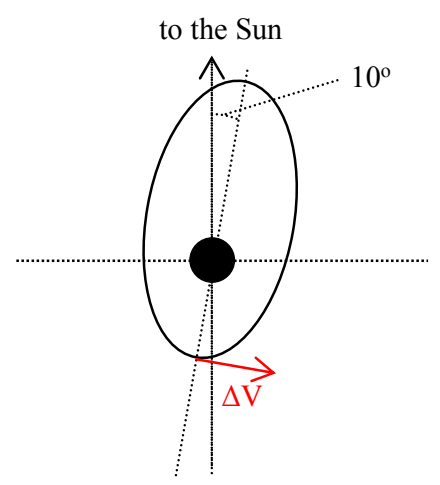

Figure 6. Initial GTO

In this study, the GTO into which the Wideband Inter-Networking engineering test and Demonstration Satellite, WINDS of JAXA, has been injected, is 
assumed to be the initial GTO of the primary payload. The PLUS2 is initiated slightly before perigee to inject the secondary payload into EGO. The departure $C_{3}$ is almost zero. Figure 6 shows the initial GTO in this study.

During the EGO flight, the on-board IES accelerates the spacecraft. The relative velocity at the Earth reencounter increases up to about $2.7 \mathrm{~km} / \mathrm{sec}$ after 1.3 year EGO flight. Figure 7 shows the EDVEGA trajectory in the inertial frame.

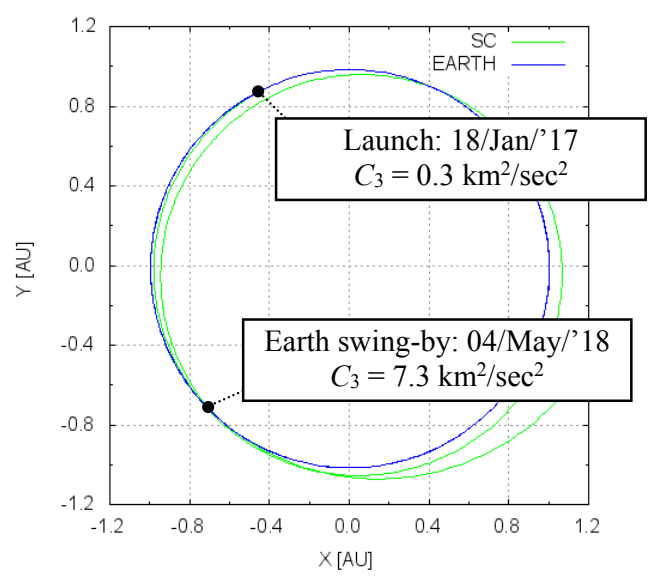

Figure 7. 1.3 year EDVEGA Trajectory in the Heliocentric Inertial Frame

Table 1 shows the specification of the on-board IES, which is almost equivalent to the IES of HAYABUSA-2 of JAXA' ${ }^{6}$.

Table 1. Specification of IES of Cases 2

\begin{tabular}{ccc}
\hline Terms & Unit & Values \\
\hline$I_{s p}$ & $\mathrm{sec}$ & 3000 \\
$F$ & $\mathrm{mN}$ & 30 \\
\hline
\end{tabular}

The specific impulse, $I_{s p}$ and thrust force, $F$, change depending on the heliocentric distance of the spacecraft designated as $R$.

Here, we assume that the thrust force $F$ and the specific impulse $I_{s p}$ are proportional to the generated power $P$ as the following ${ }^{11}$;

$$
\begin{gathered}
F=k_{1} P^{\alpha} \\
I_{s p}=k_{2} P^{\beta}
\end{gathered}
$$

where $k_{1}$ and $k_{2}$ are constants and $\alpha$ and $\beta$ are parameters. On the other hand, it is approximated that the power $P$ is proportional to the product of $F$ and $I_{s p}$ as the following;

$$
P=k_{3} F \cdot I_{s p}=k_{1} k_{2} k_{3} P^{\alpha+\beta}
$$

where $k_{3}$ is constant. Consequently, the following relationship is revealed;

$$
\beta=1-\alpha
$$

When we describe the generated power at $1 \mathrm{AU}$ as $P_{1}$, the following relationship is derived;

$$
\frac{P}{P_{1}}=\left(\frac{1 A U}{R}\right)^{2}
$$

From Eqs. (10), Eqs. (11), Eqs. (13) and Eqs. (14), the following equations are derived;

$$
\begin{gathered}
\frac{F}{F_{1}}=\left(\frac{P}{P_{1}}\right)^{\alpha}=\left(\frac{1 A U}{R}\right)^{2 \alpha} \\
\frac{I_{s p}}{I_{s p 1}}=\left(\frac{P}{P_{1}}\right)^{1-\alpha}=\left(\frac{1 A U}{R}\right)^{2-2 \alpha}
\end{gathered}
$$

where $F_{1}, I_{s p 1}$ are the thrust force and specific impulse at $1 \mathrm{AU}$, respectively. In this study, the parameter $\alpha$ is set to be $0.5^{11}$. Consequently, the following equation is derived;

$$
\frac{I_{s p}}{I_{s p 1}}=\frac{F}{F_{1}}=\frac{1 A U}{R}
$$

\section{METHOD OF ANALYSIS}

IV.I Analysis of Case 1

To evaluate and compare the gravity losses of "1Burn" and "2-Burn", the optimal burning duration and burning start point must be determined. For this purpose, Sequential Quadratic Programming, SQP, method is used in this study", ${ }^{7,}$. At first, the SQP algorithm for "1Burn" simulation is described as the following:

$\underline{\text { SQP algorithm (1-Burn) }}$

1) Control parameters $\Delta t, \Delta \varphi, \tau$

2) Equality constraint $\boldsymbol{r}_{\boldsymbol{S C}}=\boldsymbol{r}_{\boldsymbol{M}}$ at arrival epoch

3) Inequality constraint $\Delta t>0.0, \tau>0.0$

4) Objective function minimize $m_{p}$ 
where, $\tau$ is the time of flight from the Earth to Mars, $r_{S C}$ and $r_{M}$ are the 2-dimensional position vectors of the spacecraft and the Mars in the heliocentric reference frame, respectively. The total $\Delta \mathrm{V}$ of the finite time thrust, designated as $\Delta V_{\text {finite }}$ is described as the follows from Eqs. (3) and Eqs. (5):

$$
\Delta V_{\text {finite }}=g I_{s p} \ln \left(\frac{m_{0}}{m_{0}-|\dot{m}| \Delta t}\right)
$$

The gravity loss of the finite time thrust is defined as follows ${ }^{4}$ :

$$
\text { gravity loss } \approx \frac{\Delta V_{\text {finite }}}{\Delta V_{\text {impulse }}}-1
$$

where the $\Delta V_{\text {impulse }}$ is computed by Eq. (7) and the initial $\Delta t$ and $\Delta \varphi$ are determined by Eqs. (8) and (9). The initial $\tau$ is 200 days.

On the other hand, the SQP algorithm for "2-Burn" simulation is the following:

$\underline{\text { SQP algorithm (2-Burn) }}$

1) Control parameters

$$
\Delta t_{1}, \Delta t_{2}, \Delta \varphi_{1}, \Delta \varphi_{2}, \tau
$$

2) Equality constraint $\boldsymbol{r}_{S C}=\boldsymbol{r}_{\boldsymbol{M}}$ at arrival epoch

3) Inequality constraint $\Delta t_{1}>0.0, \Delta t_{2}>0.0, \tau>0.0$

4) Objective function minimize total $m_{p}$

where the subscript numbers correspond to $\Delta V_{1}$ or $\Delta V_{2}$.

\section{IV.II Analysis of Case 2}

In the Case 2 study, the optimal EGO is computed by minimizing the departure $\Delta \mathrm{V}$ which is applied at the perigee point of the initial GTO. For this simulation, an initial EGO is determined in advance through heuristic approach by changing the time of flight during the EGO phase $\tau_{E G O}, \Delta \mathrm{V}$ at perigee $\Delta V_{p}$, IES throttle level $T L$, and IES thrust unit vector, $\boldsymbol{F}_{I E S}$. $V_{\infty} O$ at the Earth reencounter is also determined from the trajectory design between the Earth and Mars. Once the initial trajectory is determined, the EGO is divided into 48 nodes, and the parameters are input to the following SQP method:

$\underline{\text { SQP algorithm (Case 2) }}$

1) Control parameters $\Delta V_{p}, \tau_{E G O}, T L_{n}, \boldsymbol{F}_{I E S n}$
2) Equality constraint $R_{S C-E}=R_{S O I}$ at Earth re-encounter $\left|V_{\infty I}\right|=\left|V_{\infty O}\right|$ at Earth re-encounter

3) Inequality constraint

$0.0 \leq T L_{n} \leq 1.0$

$200(\mathrm{~km}) \leq$ Alt $_{s b}$

4) Objective function minimize $\Delta V_{p}$

where $R_{S C-E}$ is the range between the spacecraft and the Earth at the Earth re-encounter, $R_{S O I}$ is the radius of the sphere of influence of the Earth, $V_{\infty I}$ and $V_{\infty O}$ are the inbound and out-bound relative velocities at the Earth reencounter, and $A l t_{s b}$ is the altitude at the Earth swing-by. The subscript $n$ attached to $T L$ and $\boldsymbol{F}_{\text {IES }}$ is the node number.

The radius of the Earth's sphere of influence, $R_{S O I}$, is described as follows ${ }^{5}$ :

$$
R_{S O I}=\left(\frac{M_{E}}{M_{S}}\right)^{\frac{2}{5}} A_{E}
$$

where $M_{E}$ and $M_{S}$ are the mass of the Earth and Sun, respectively, and $A_{E}$ is the heliocentric distance of the Earth.

When the turning angle $\Phi$, which is the angle between $V_{\infty I}$ and $V_{\infty O}$ at the Earth swing-by are known, the range between the Earth and spacecraft at the Earth swing-by, $r_{S C-E}$, is described as follows ${ }^{3,5}$;

$$
r_{S C-E}=-\frac{\mu_{E}}{V_{\infty}^{2}}\left(1+\frac{1}{\cos f_{\infty}}\right)
$$

where;

$$
\Phi=2 f_{\infty}-\pi
$$

The $A t_{s b}$ is determined by subtracting the Earth's radius from $r_{S C-E}$.

Note that the on-board IES is initiated 3 days after launch. Besides, the IES is activated during only EGO phase i.e. the IES is not used during and after the Earth swing-by.

\section{RESULTS OF ANALYSIS}

\section{V.I Results of Case 1 Analysis}

Table 2 summarizes the specification of PLUS1 of Case $1 . m_{s}$ is the mass of PLUS1 structure, $m_{p}$ is the propellant mass, $m_{p l}$ is the payload mass, $\eta$ is the propellant mass fraction, and $F$ is the thrust force of PLUS1. 
Table 2. Specification of PLUS1 of Case 1

\begin{tabular}{ccc}
\hline Terms & Unit & Values \\
\hline$m_{0}$ & $\mathrm{~kg}$ & 17363 \\
$m_{s}$ & $\mathrm{~kg}$ & 1867 \\
$m_{p}$ & $\mathrm{~kg}$ & 12496 \\
$m_{p l}$ & $\mathrm{~kg}$ & 3000 \\
$\eta$ & -- & 0.87 \\
$I_{s p}$ & $\mathrm{sec}$ & 340 \\
$F$ & $\mathrm{kN}$ & 29.4 \\
\hline
\end{tabular}

Table 3 summarizes the keplerian elements at the initial epoch i.e. 00:00, May, $4^{\text {th }}, 2018$ (UTC). In this analysis, the parking orbit lies on the ecliptic plane i.e. the inclination is $23.4 \mathrm{deg}$. In Table $3, a$ is the semimajor axis, $e$ is the eccentricity, $i$ is the inclination, $\Omega$ is the right ascension of the ascending node, $\omega$ is the argument of perigee and $v$ is the true anomaly. Note that the Sun-Earth line is inclined $40 \mathrm{deg}$ from the $+\mathrm{X}$ direction of the heliocentric reference frame at the initial epoch. The simulation starts from the Sun-Earth line, hence the true anomaly $v$ at the initial epoch is $40 \mathrm{deg}$.

Table 3. Keplerian Elements of Parking Orbit

\begin{tabular}{ccc}
\multicolumn{3}{c}{ at Initial Epoch } \\
\hline Term & Unit & Values \\
\hline$a$ & $\mathrm{~km}$ & 6678.0 \\
$e$ & -- & 0.0 \\
$i$ & $\operatorname{deg}$ & 23.4 \\
$\Omega$ & $\mathrm{deg}$ & 0.0 \\
$\omega$ & $\operatorname{deg}$ & 0.0 \\
$v$ & $\mathrm{deg}$ & 40.0 \\
\hline
\end{tabular}

The results of Case 1 analysis are summarized in Table 4.

Table 4. Results of Case 1 Analysis

\begin{tabular}{cccc}
\hline Term & Unit & 1 -Burn & 2 -Burn \\
\hline$\Delta V_{\text {impulse }}$ & $\mathrm{km} / \mathrm{s}$ & 3.505 & 3.503 \\
$\Delta V_{\text {finite }}$ & $\mathrm{km} / \mathrm{s}$ & 3.662 & 3.547 \\
$\Delta \varphi_{1}$ & $\mathrm{deg}$ & 42.1 & 26.4 \\
$\Delta \varphi_{2}$ & $\mathrm{deg}$ & -- & 5.6 \\
$\Delta t_{1}$ & $\mathrm{sec}$ & 1311.6 & 808.8 \\
$\Delta t_{2}$ & $\mathrm{sec}$ & -- & 480.1 \\
$\tau$ & $\mathrm{day}$ & 196 & 191 \\
residual $m_{p}$ & $\mathrm{~kg}$ & 923 & 1125 \\
gravity loss & $\%$ & 4.480 & 1.256 \\
\hline
\end{tabular}

From the results of Table 4, the gravity loss apparently improves by applying the 2-Burn method. The difference in residual mass of the propellant between 1-Burn and 2-Burn is about $200 \mathrm{~kg}$.

As mentioned, from a gravity loss perspective, the "2-Burn" case is superior, however, in the "2-Burn" case, PLUS1 is additionally required to reignite after one revolution in the intermediate orbit i.e. 4.3 hours.
Table 5. shows the keplerian elements at the $\Delta V_{2}$ burning start point.

Table 5. Keplerian Elements of Intermediate Orbit at $\Delta V_{2}$ Burning Start Point

\begin{tabular}{ccc}
\hline Term & Unit & Values \\
\hline$a$ & $\mathrm{~km}$ & 13418.3 \\
$e$ & -- & 0.5 \\
$i$ & $\mathrm{deg}$ & 23.4 \\
$\Omega$ & $\mathrm{deg}$ & 0.0 \\
$\omega$ & $\mathrm{deg}$ & 157.5 \\
$v$ & $\mathrm{deg}$ & 341.5 \\
orbital period & $\mathrm{hr}$ & 4.3 \\
\hline
\end{tabular}

Note that the argument of perigee $\omega$ is 157.5 which is $117.5 \mathrm{deg}$ from the Sun-Earth line. Thus the angle $\delta / 2$ is $62.5 \mathrm{deg}$ which is almost equivalent to the value computed by Eqs. 1 i.e. 64.0 deg (See Figure 4).

\section{V.II Results of Case 2 Analysis}

Table 6 summarizes the specification of the PLUS2 of Case 2.

Table 6. Specification of PLUS2 of Case 2

\begin{tabular}{ccc}
\hline Terms & Unit & Values \\
\hline$m_{0}$ & $\mathrm{~kg}$ & 800 \\
$m_{s}$ & $\mathrm{~kg}$ & 90 \\
$m_{p}$ & $\mathrm{~kg}$ & 210 \\
$m_{p l}$ & $\mathrm{~kg}$ & 500 \\
$\eta$ & -- & 0.7 \\
$I_{s p}$ & $\mathrm{sec}$ & 340 \\
$F$ & $\mathrm{kN}$ & 9.8 \\
\hline
\end{tabular}

The H-2A 204 has the ability to launch a $6000 \mathrm{~kg}$ payload into GTO. Accordingly, if the mass of the primary payload is less than $5000 \mathrm{~kg}$, an $800 \mathrm{~kg}$ secondary payload, consisting of a $500 \mathrm{~kg}$ small explorer and a $300 \mathrm{~kg}$ PLUS2, can be launched together with the primary payload.

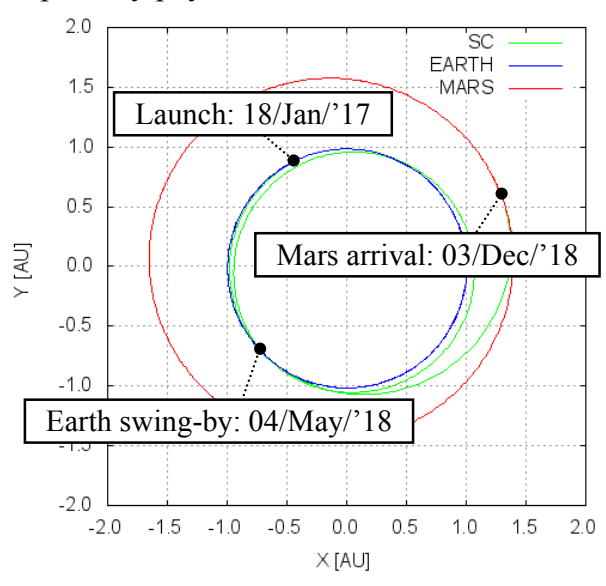

a) Heliocentric Reference Frame 


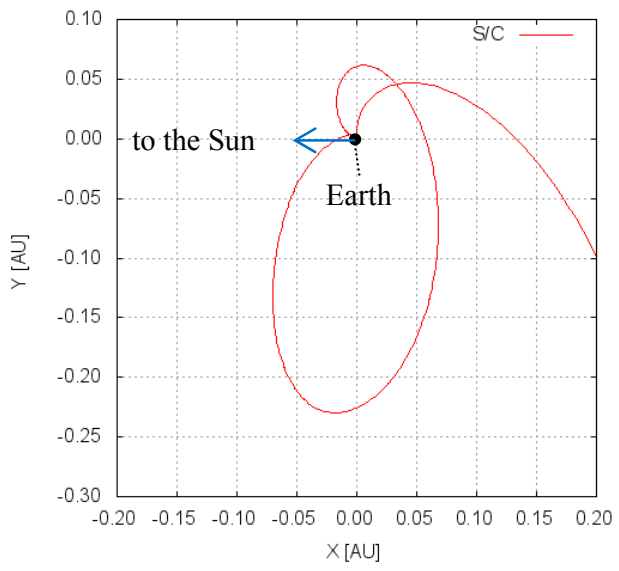

b) Geocentric Rotational Frame

Figure 8. EDVEGA and Mars Transfer Trajectory

Figure 8 shows the EDVEGA and Mars transfer trajectory optimized by the SQP algorithm in the heliocentric reference frame and geocentric rotational frame.

Table 7 summarizes the result of Case 2 analysis. Based on the results summarized in Table 7, PLUS2 system assumed as shown in Table 6 does work for the secondary payload injection into EGO from the initial GTO.

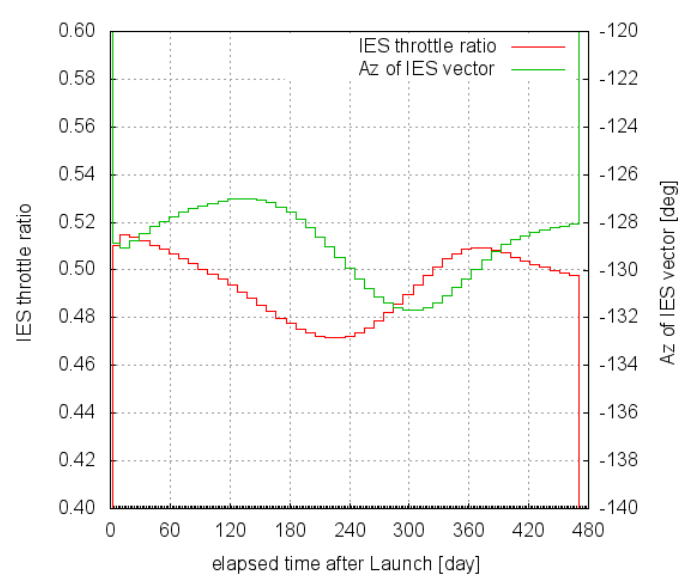

Figure 9. Time Variation of IES Throttle Level and Thrust Vector

Figure 9 shows the time variation of IES throttle level and IES thrust vector in the heliocentric reference frame. As shown in the figure, the IES thrust vector is almost constant in the heliocentric reference frame during the EGO phase. The mass of propellant consumed by the IES is $41.2 \mathrm{~kg}$ while the propellant of HAYABUSA is $66 \mathrm{~kg}^{12}$.

Table 7. Results of Case 2 Analysis

\begin{tabular}{ccc}
\hline Terms & Unit & Values \\
\hline$\Delta V_{p}$ & $\mathrm{~m} / \mathrm{s}$ & 796.8 \\
$\Delta t$ & $\mathrm{sec}$ & 57.9 \\
$m_{p}$ consumed & $\mathrm{kg}$ & 170 \\
$\tau_{E G O}$ & day & 470 \\
\hline
\end{tabular}

Launch Window Expansion

In the type of use of Case 2, the secondary payload cannot choose the "desired" launch epoch. The launch windows of deep-space missions are generally narrow i.e. 1 2 weeks, hence a method of expanding the launch window of the secondary payload i.e. small explorer is necessary for the Case 2 use. The study on the flexible orbit design technique which enables "any-time" launch of the deep-space explorer is under way. According to the results of the preliminary study, the launch window can be drastically widened i.e. 360 days ${ }^{10}$.

\section{CONCLUSION}

This study assessed the launch capability of the optional kick stage, PLUS (for Planetary, Long-duration Upper Stage) system through numerical simulations.

In Case 1 study, it was assumed to launch a $3000 \mathrm{~kg}$ explorer to Mars by PLUS system designated as "PLUS1". The thrust force of PLUS1 is relatively small i.e. $29.4 \mathrm{kN}$ however split $\Delta \mathrm{Vs}$ improved the performance efficiency to practical level i.e. $1.3 \%$ gravity loss, while the loss of "1-Burn" case is $4.5 \%$. This improvement means about $200 \mathrm{~kg}$ propellant is saved by the split $\Delta \mathrm{Vs}$, although PLUS1 is required to reignite at 4.3 hours after the first $\Delta \mathrm{V}$.

In Case 2 study, on the other hand, it was assumed that the secondary payload consisting of a $500 \mathrm{~kg}$ explorer and small PLUS system, designated as "PLUS2", is dual launched into GTO together with a $5000 \mathrm{~kg}$ primary payload. On the perigee point of the GTO, PLUS2 is ignited to inject the explorer into the trajectory whose orbital energy, $C_{3}$, is almost zero. After the injection, the explorer voyages to Mars through EDVEGA scheme via the on-board IES. The numerical simulation revealed a HAYABUSA class explorer can be launched as a secondary payload into GTO, and also the explorer can reach Mars after 1.3 year energy garnering phase.

\section{REFERENCES}

1) “Proton Launch System Mission Planner's Guide, LKEB-9812-1990”, SECTION 2, LV Performance, Revision 7 July, 2009 
2) Kawaguchi. J., "On the Delta-V Earth Gravity Assist Trajectory (EDVEGA) Scheme with Applications to Solar System Exploration", 52 nd International Astronautical Congress, 1-5 Oct, 2001/Toulouse, France, 2001

3) Utashima. M., "Orbital Design to Ulysses-type Solar Polar Orbit by Solar Electric Propulsion System (in Japanese)", NASDA Technical Memorandum, NASDA-TMR-010021, 2002

4) Giacomo Porcelli, "Suboptimal, Two-burn, Midlevel Thrust, LEO-GEO Transfer: Practical Control Schemes", Acta Astronautica Vol. 12, No. 10, pp. 847-852, 1985

5) John E. Prussing, Bruce A. Conway, "Orbital Mechanics”, Oxford University Press, 1993

6) Tsuda. Y., Yoshikawa. M., Abe. M., Minamino. H., Nakazawa. S., "System Design of Hayabusa2 - Asteroid Sampe Return Misson to 1999JU3", 63 ${ }^{\text {rd }}$ International Astronautical Congress, IAC-12-A.3.4.5, 2012

7) Bazaraa.M. S., Sherali. H. D., Shetty. C. M., "Nonlinear Programming Theory and Algorithms Third Edition", WILEY-INTERSCIENCE, 2005

8) NLopt web page, "http://ab-initio.mit.edu/wiki/index.php/NLopt"

9) James R. Wertz, Wiley J. Larson, "Space Mission Analysis and Design Third Edition”, Space Technology Library, 1999

10) Ikenaga. T., Utashima. M., Ishii. N., Yoshikawa. M., Hiraiwa. T., Noda. A., “A study on a low-cost deep space exploration utilizing a small kick stage (in Japanese)", U07-P13, Japan Geocentric Union Meeting, 2013

11) Utashima. M., "Orbital Design for Ulysses-type Solar Polar Orbit by H-2A (in Japanese)", NASDA Technical Memorandum, NASDA-TMR-010011, 2001

12) Kuninaka. H., Nishijima. K., Funaki. I., Yamada. T., Shimizu. Y., Kawaguchi. J., "Powered Flight of HAYABSUA in Deep Space", 42 ${ }^{\text {nd }}$ AIAA/ASME/SAE/ASEE Joint Propulsion Conference \& Exhibit, AIAA 2006-4318, 2006 